Classification

Physics Abstracts

$71.25 \mathrm{Tn}-78.90+\mathrm{t}$

\title{
The interpretation of near edge structure
}

\author{
Peter $\operatorname{Rez}\left({ }^{1,2}\right)$, Xudong Weng $\left({ }^{1,3}\right)$ and Hong Ma $\left({ }^{1}\right)$ \\ ( $\left.{ }^{1}\right)$ Center for Solid State Science, Arizona State University, Tempe, AZ 85287-1704, U.S.A. \\ ( ${ }^{2}$ ) Department of Physics, Madingley Road, Cambridge CH3 OHE, England \\ $\left({ }^{3}\right)$ Cavendish Laboratory, Madingley Road, Cambridge CH3 OHE, England
}

(Received November 09, 1990; accepted March 26, 1991)

\begin{abstract}
The near edge structure up to $30 \mathrm{eV}$ above the ionisation threshold of inner shell edges can be interpreted either as the result of interference of waves scattered from neighbouring atoms or in terms of the local projected conduction band density of states. Methods of calculating near edge structure based on both these viewpoints will be discussed and applications to the near edge structures observed in semiconductors and insulators reviewed. Particular emphasis will be given to those cases where the calculations lead to a simple interpretation of near edge structure.
\end{abstract}

The characteristic inner shell edges observed in electron energy loss spectroscopy have traditionally been used for elemental analysis. Now that spectrometers capable of giving about 1 to $1.5 \mathrm{eV}$ resolution on conventional TEMs and 0.3 to $0.5 \mathrm{eV}$ resolution on cold field emission STEMs have become available the observation of fine structure oscillations has become routine. In particular the large modulations within the first 20 to $30 \mathrm{eV}$ above threshold have become easy to observe and can be detected even when the element is present in low concentrations (below about $10 \%$ relative to the majority constituent). It would be advantageous if the fine structures could give some new information on the microstructure of the material, especially if that information could not be obtained in other ways. The materials scientist would like to know whether the near edge structure gives information about elemental charge state, local bonding or the local nature of the conduction band. Alternatively the fine structure might be sensitive to environment or coordination number. Another possibility is that it only reflects interatomic bond distances, but in a more convoluted way than the much weaker extended fine structure which starts about $30 \mathrm{eV}$ above threshold.

One approach to understanding near edge structure is to collect "fingerprints" from elements in similar environments and try to identify common features (see the article by Brydson in this volume). The other approach, which is adopted in this paper, is to calculate the near edge structure using appropriate theory and identify those features which show sensitivity to a property of interest. In practice both methods can be valuable and they readily complement each other.

Calculations have traditionally relied on two theoretical approaches. The most common method, which has been extensively used by the X-ray absorption community, is the cluster calculation or XANES method of Durham et al. (1982) [1]. The key idea is that the fine structure 
arises from the interference of electron waves scattered from neighbouring atoms and the outgoing wave representing the ejected inner shell electron. This is, of course, the basis of the weak extended fine structure (EXAFS or EXELFS) but the significant difference is that all orders of multiple scattering within a cluster defined by a given number of atomic shells around the excited atom is included.

The other approach relies on the fact that the differential cross section for inelastic scattering, $\mathrm{d}^{2} I$ $\frac{\mathrm{d}^{2} I}{\mathrm{~d} \Omega \mathrm{d} E}$, is a product of the square of some matrix element $M(E)$ and an appropriate density of final states, $\rho(E)$.

$$
\frac{\mathrm{d}^{2} I}{\mathrm{~d} \Omega \mathrm{d} E} \propto|M(E)|^{2} \rho(E)
$$

The density of states can then be calculated by normal band theory techniques. Although the methods appear quite different the XANES method is no more than a local, real space, variation on the KKR band theory technique in which a Bloch condition has not been imposed.

\section{Cluster calculations of near edge structure in oxides.}

In the XANES method as originally developed by Durham et al. (1981) [1] the crystal is divided into spherical atomic regions called muffin tins. The region between the muffin tins is set at a constant potential (the muffin tin zero). The potential inside the muffin tins is constructed using the atomic potential from a Hartree-Fock-Slater calculation [2]. The spherically symmetric parts of the potential from neighbouring atoms is superposed using the prescription suggested by Mattheis (1964) [3]. Scattering from these atomic muffin tins is represented by the phase shifts at the muffin tin radius which are obtained by matching the solution of ingoing and outgoing spherical waves to the solution of the Schrodinger equation for an electron of the appropriate energy above the muffin tin zero. In this case the muffin tin zero represents the inner shall threshold. Exchange is treated by the $X_{\alpha}$ prescription which assumes that the exchange potential is a function of the local electron density. In the XANES method the scattering from the various shells is summed first and then the various scattering events between the shells is considered. In this way a separation can be made between the intrashell scattering and the intershell scattering.

A method closely related to the XANES method has been developed by Fujikawa (1983) [4]. The significant difference is that he does not sum over the scattering within a coordination shell before calculating the intershell processes. The Slater and Johnson (1972) [5] $\mathrm{X}_{\alpha}$ method is also closely related to the XANES method though in its original form it was mainly used to provide energies of molecular orbitals. The major difference between the $\mathrm{X}_{\alpha}$ method and the XANES method is that the excited electron wave function is used to recalculate the charge density, and hence the potential, until self consistency is reached. In the XANES method the excited electron is assumed to scatter in a one-electron potential that has already been defined. Another less significant difference is that the $\mathrm{X}_{\alpha}$ method allows for an outgoing spherical wave travelling away from the cluster.

All the methods as described above are one-electron schemes in that no attempt is made to consider the effects of the core hole on the remaining electrons. There have been attempts to incorporate the core hole effect in cluster schemes using an atom of atomic number $Z+1$ for the excited atom [6], or by using a transition state in which phase shifts are calculated with fractional occupancy in the inner shell and some higher shell.

We were interested in determining whether the near edge structure could be used to determine ionicity as reported by Oizumi et al. (1985) [7] in a study in alkali halides. We applied the XANES method to the calculation of the near edge structure on the oxygen $\mathrm{K}$ edge of various oxides with 
the $\mathrm{NaCl}$ structure (see Fig. 1). The structures all show three peaks in the region up to $30 \mathrm{eV}$ and we varied the charge in the muffin tins to see whether there was significant variation. In fact we obtained the best agreement with neutral atom muffin tins (see Fig. 1) though the difference between these results and those for ions with charge 2+ and 2- was small. In the case of $\mathrm{MgO}$ our result is very similar to that of Lindner et al. (1986) [6] and in good agreement with experiment even though we did not attempt to take into account the core hole using the $Z+1$ approximation.

An examination of the breakdown of the fine structure contributions from the various shells (Fig. 2) gives a simple explanation of the origin of the various features. In these structures the metal cations scatter weakly and only the strong scattering from the oxygen anions need be considered. In figure 1 the third peak $(C)$ is the result of single scattering (EXAFS) from the nearest neighbour oxygen shells. The second peak (B) arises from single (EXAFS) scattering from the 4th shell around the excited atom (second oxygen shell). The first large peak (A) comes from a triple scattering involving an intrashell scattering in the first neighbouring oxygen shell. Accordingly one would predict that if the material were to become disordered the second peak would become broader and eventually vanish. One would also expect that the higher the coordination number the higher the first peak relative to the second two peaks. Although these effects have not been observed in oxides they are consistent with variations in Aluminium and Silicon $\mathrm{K}$ shell near edge structure in environments with differing coordination [8]. Using these arguments and approximate values of the phase shifts it is possible to show that the peak positions are consistent with this simple idea. It would therefore appear that in the case of strong scatterers the height of intrashell peaks near threshold when compared to single scattering EXAFS peaks should give a measure of coordination.

\section{Band theory methods.}

The basic idea behind the band theory methods is that the excited electron is probing the density of unoccupied states multiplied by the square of the matrix element. In a region 10 to $20 \mathrm{eV}$ above threshold the matrix elements vary relatively slowly (about a $20 \%$ decrease from threshold for a typical $\mathrm{K}$ shell) and most of the structure observed is a direct manisfestation of density of states variation. Equation (1), as stated above, takes no account of the selection rules for inelastic scattering. For small collection angles the dipole approximation is quite acceptable. Under dipole scattering conditions X-ray and energy loss spectra are identical. The final state angular momentum quantum number $L^{\prime}$ is then given by $L+1$ or $L-1$ where $L$ is the initial state quantum number. In general the $L+1$ scattering channel is more significant than the $L-1$ channel. The differential cross section should be written as

$$
\frac{\mathrm{d}^{2} I}{\mathrm{~d} \Omega \mathrm{d} E}=\frac{4 \gamma^{2}}{a_{0}^{2} q^{2}}\left[\left|m_{L+1}\right|^{2} \rho_{L+1}(E)+\left|m_{L-1}\right|^{2} \rho_{L-1}(E)\right]
$$

where $m_{L+1}$ is the matrix element for transitions to states of angular momentum $L+1$ and $\rho_{L+1}$ is the density of states of the $L+1$ angular momentum. Similar definitions in terms of $L-1$ hold for the second term. It is apparent that what is required is the appropriate angular momentum resolved density of states at the site of the excited atom (a projected density of states). Not all band theory methods can make this readily available.

Some of the best results have been given by the Linearised Augmented Plane Wave (LAPW) method or the Augmented Spherical Wave method [9]. The LAPW program has been applied to transition metals and their carbides, oxides and nitrides [10]. It is particularly well suited to computations involving the $\mathrm{d}$ bands of these materials. Normally the pseudopotential band theory techniques, which have been so successful when applied to semiconductors, have not been 

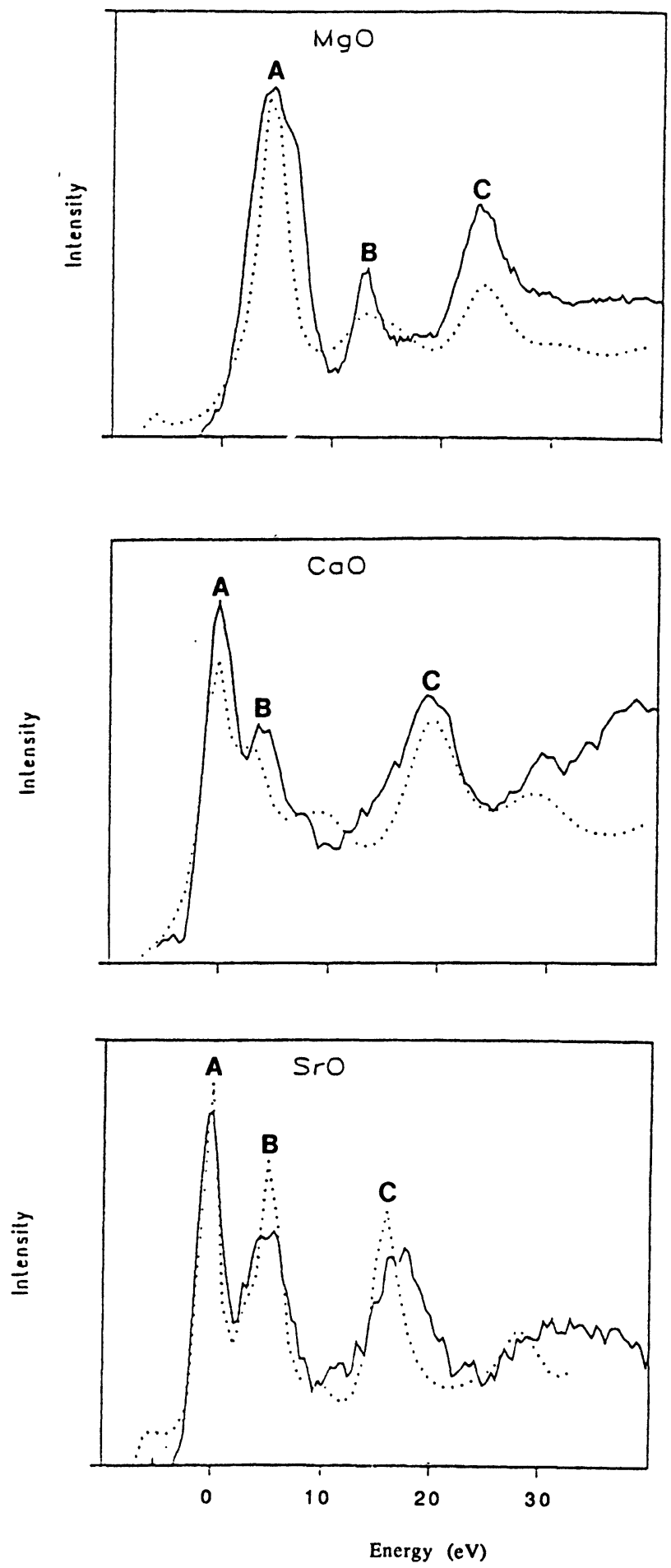

Fig. 1. - Oxygen $\mathrm{K}$ edge structure for (a) $\mathrm{MgO}$, (b) $\mathrm{CaO}$, (c) SrO. The experimental spectrum is shown as a solid line, the XANES calculation result by a dotted line. 


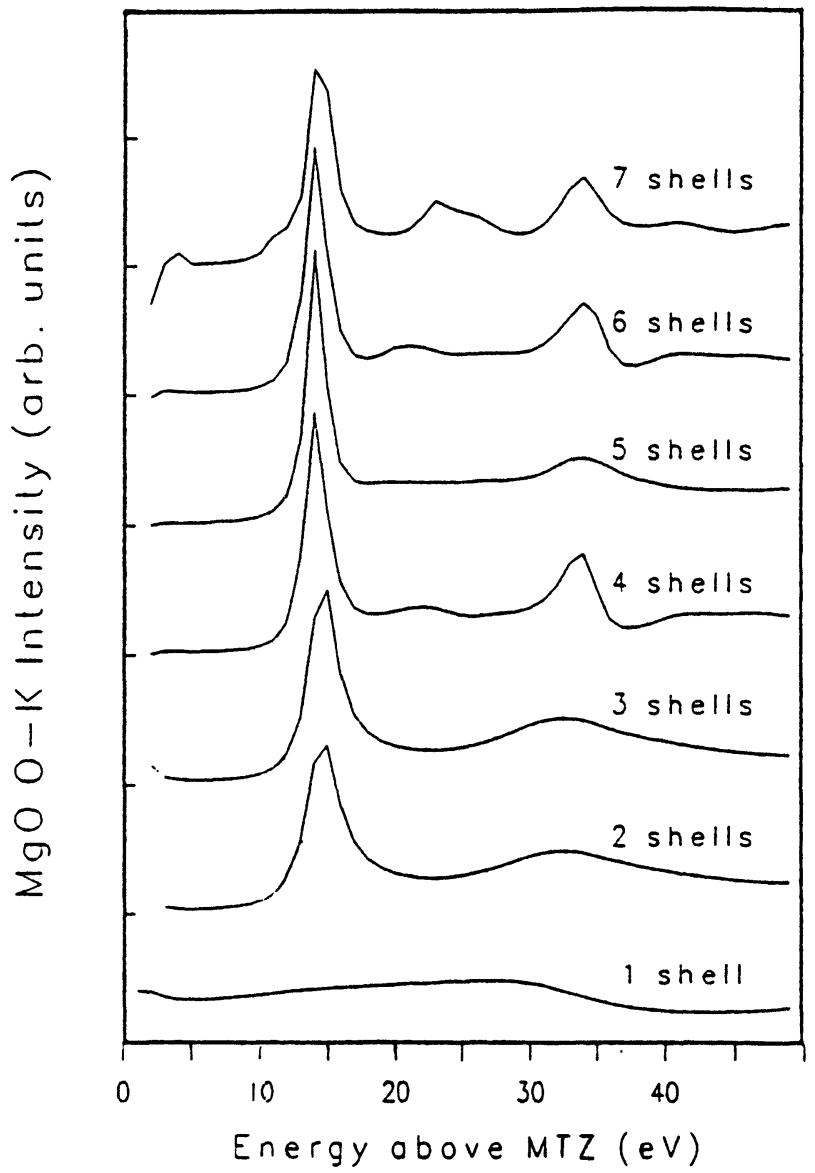

Fig. 2. - Oxygen $\mathrm{K}$ edge fine structure for $\mathrm{MgO}$ as a function of cluster size in the XANES calculation.

applied to this problem as they use plane waves as a basis set and these have not been projected in the appropriate way. We have used a variation of the pseudo-potential method in which the basis of the expansion are pseudo atomic orbitals calculated from a sum of Slater-type orbitals [11]. The decomposition into states of different angular momentum is therefore explicit in the formalism. Bloch functions can be constructed from these states and an appropriate Hamiltonian diagonalised to yield the energy bands. The Hamiltonian uses a self consistent pseudopotential with the local density approximation (LDA) [12] for correlation and exchange. This means that the electron exchange and correlation parts of the effective potential can be expressed as functions of the local electron density. Strictly the local density approximation does not apply to empty states, but to go beyond this approximation is computationally very difficult. In the end we can justify our use of the LDA by the agreement with experiment. The densities of states can be calculated from the usual prescription by integrating the reciprocal of the energy gradient in $\mathrm{K}$ space over constant energy surfaces.

$$
\rho(E)=\int_{S_{n}(E)} \frac{1}{\left|\nabla E_{n}(K)\right|} \frac{\mathrm{d} S}{4 \pi^{3}}
$$

We have applied this method [13] to calculate the near edge structure of elemental $\mathrm{Si}$ (K and $\mathrm{L}_{23}$ 
edges), Diamond (K edge), Beryllium Carbide (Be and Carbon Kedges) and Silicon Carbide (Si K and $\mathrm{L}_{23}$ edges and Carbon K edge). In hexagonal materials we have calculated the edge shapes for the $\mathrm{K}$ edges in both graphite [14] and hexagonal boron nitride. The results for silicon carbide are shown as figure 3 with a comparison to the experimental results of Krishnan. The agreement with experiment is very good and all the fine structure features are reproduced. Note the sharp peak at the threshold of the $\mathrm{Si}_{23}$ edge. This is due to transitions to empty s states. At threshold the $p$ to $d$ transitions are suppressed due to the centrifugal barrier. A similar effect can be observed in the high resolution work of Batson on elemental Silicon and we have previously published a comparison of our calculations with his experimental data showing excellent agreement [15]. It is important to be aware of these strong $p$ to $s$ transitions at the threshold of the $\mathrm{L}_{23}$ excitation. They should not be confused with a core exciton, though the possibility of modification by excitons can not be ruled out. From our calculation we believe that the core exciton in Si has a binding energy of no more than $50 \mathrm{meV}$ which is in agreement with the X-ray emission work of Carson and Schnatterly (1987) [16]. Another example of a comparison of calculation with experiment is given as figure 4 where we show the near edge structure of on both the Boron and Nitrogen peaks in cubic boron nitride compared with the experimental measurements of Hofer.

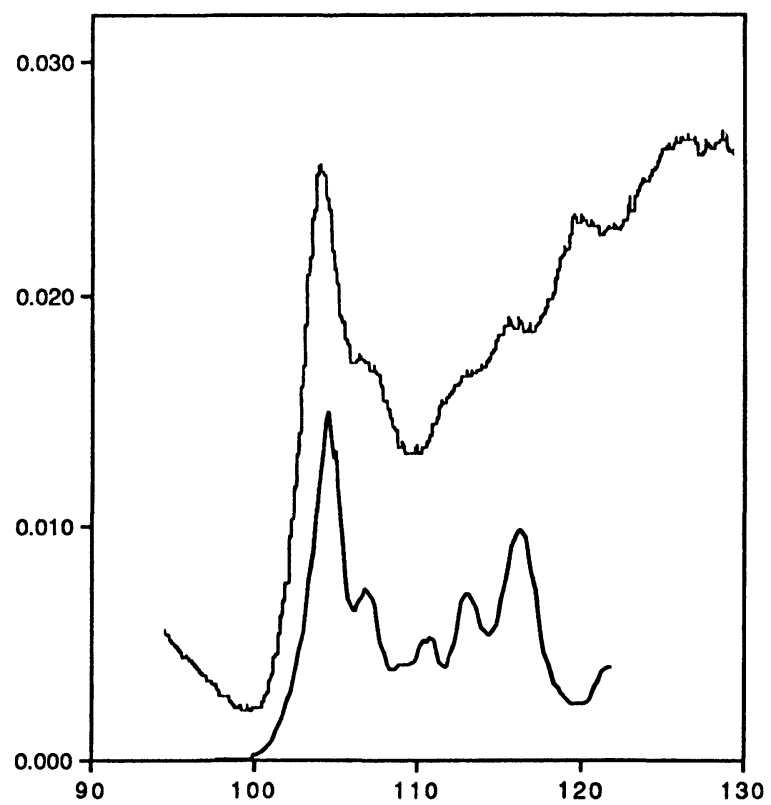

Encrgy (cV)

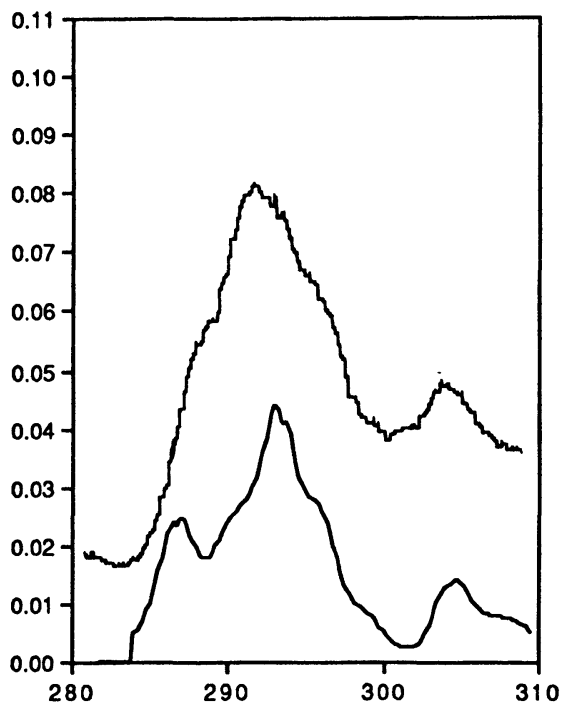

Energy (eV)

b)

Fig. 3. - (a) Silicon $\mathrm{L}_{23}$ edge in $\beta$ SiC. The lower curve is the pseudo-atomic-orbital calculation result, the upper curve is the experimental result of Krishnan and Echer. (b) Carbon $\mathrm{K}$ edge in $\beta$ SiC. The lower curve is the pseudo-atomic-orbital calculation result, the upper curve is the experimental result of Krishnan and Echer.

Apart from identification of certain peaks with molecular orbitals ( $\sigma^{*}$ and $\pi^{*}$ in the case of graphite and hexagonal boron nitride [17]) or mapping peak positions on to flat regions of the 


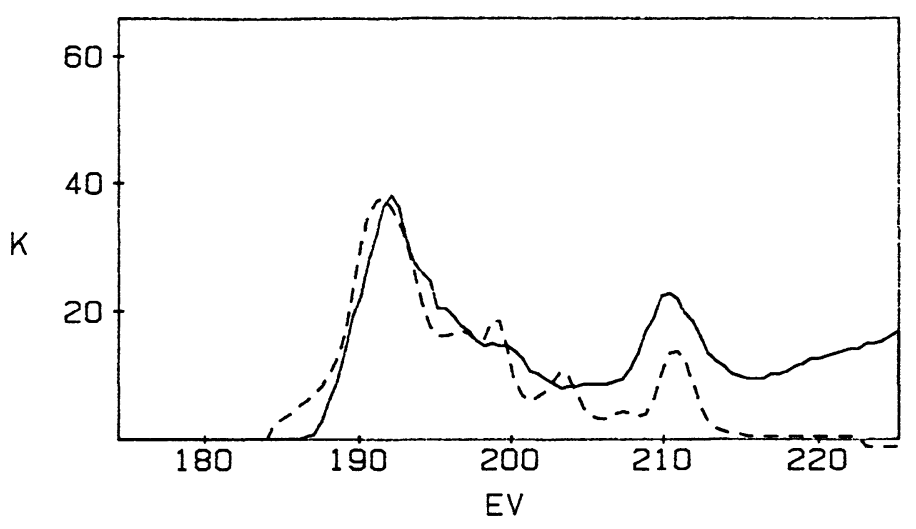

a)

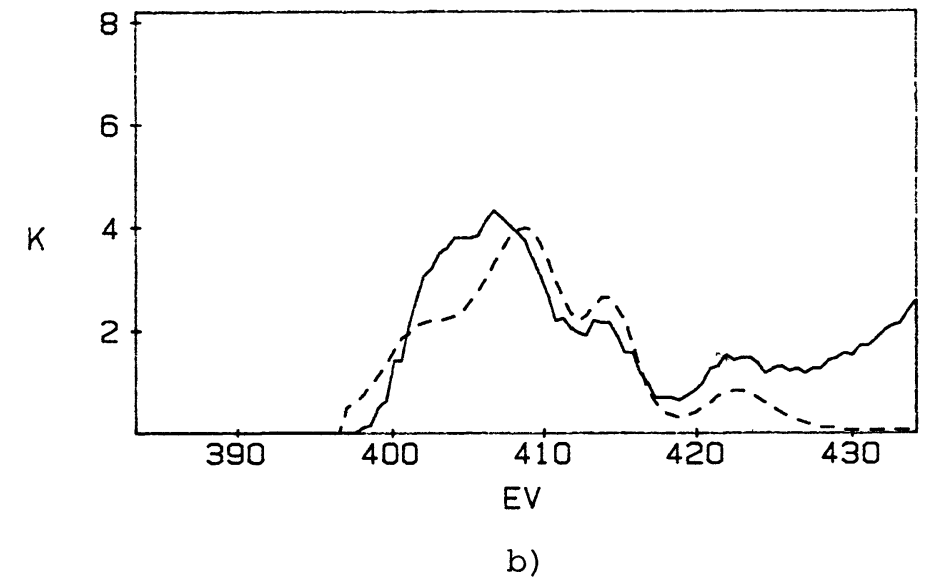

Fig. 4. - (a) Boron $\mathrm{K}$ edge in cubic BN. The dashed curve is the pseudo-atomic-orbital calculation result, the solid line is the experimental result of Hofer. (b) Nitrogen $\mathrm{K}$ edge in cubic BN. The dashed curve is the pseudo-atomic-orbital calculation result, the solid line is the experimental result of Hofer.

band structure simple interpretations are somewhat elusive. It is helpful for some clues to return to a plot of the densities of states. In silicon carbide the bonding has $\mathrm{sp}^{3}$ character and is well represented by the density of $p$ states. Figure 5 shows the $p$ density of states on both the silicon and carbon sites. In accord with naive views on electronegativity there is a large $p$ valence density of states and a small empty conduction band state density. The inverse is true for the p density of states on the Si site. This suggests that the relative magnitude of the fine structure oscillations should be a crude measure of electronegativity or charge transfer. As Si K edges and Carbon $\mathrm{K}$ edges are not usually acquired under similar conditions it has not been possible to check this hypothesis with existing data.

\section{Conclusions.}

Near edge structure can be used to give information about materials that is not usually accessible. In cases where the XANES cluster calculations do well it is sometimes possible to identify those peaks that arise single scattering and those that are due to intrashell scattering processes. 


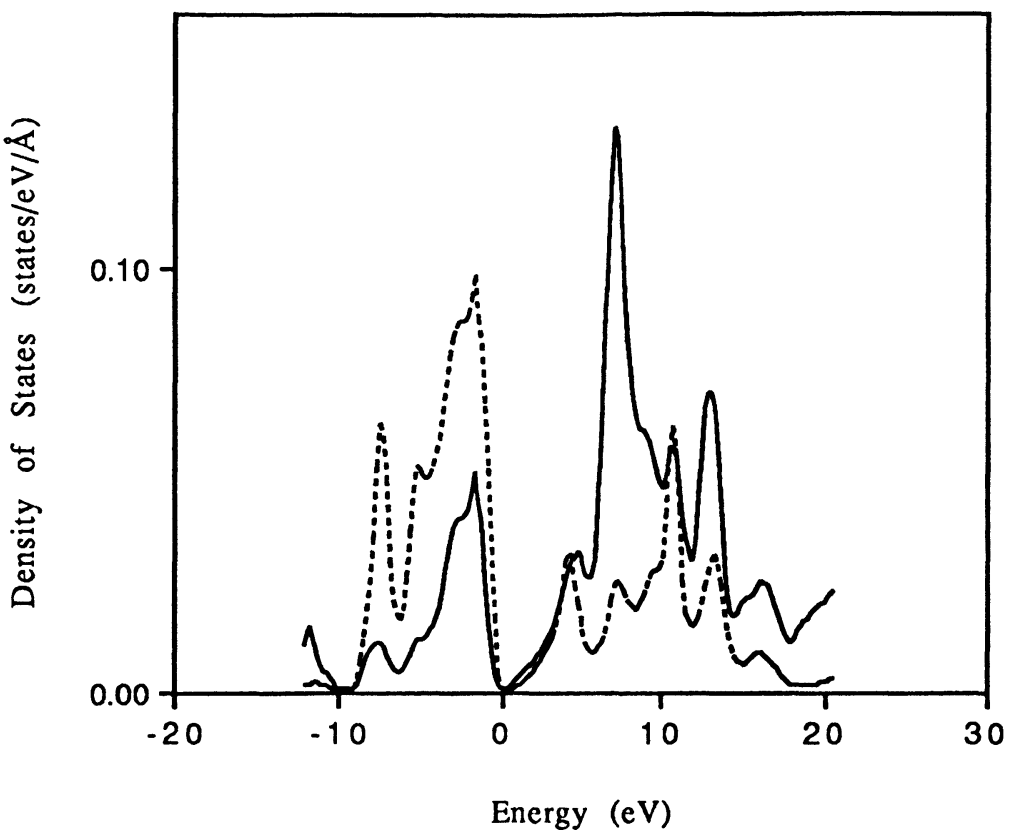

Fig. 5. - p density of states for silicon (solid line) and carbon (dashed line) in $\beta$ SiC.

Comparing the heights of these different features gives a measure of coordination by strongly scattering neighbours. In our experience the XANES method work best when there is strong scattering and the symmetry allows for an obvious partitioning into muffin tins. They also perform well when there is a dense packing. XANES calculations have been less successful for the less densely packed semiconductor structures and the results (as expected) are quite poor for layer materials such as graphite [14]. Recent work by Stohr using the $\mathrm{X}_{\alpha}$ method has shown better results than XANES for small molecular clusters. This might be attributed to the lack of self consistency in the XANES procedure.

The pseudo-atomic-orbital calculations have given very good agreement with the near edge structure in semiconductors and ceramic materials in the region up to $15 \mathrm{eV}$ above threshold. They have been shown to be adequate for interpreting the near edge structure at resolutions of up to $0.5 \mathrm{eV}$. What many electron effects should be included for a description at superior energy resolution is, at present, an open question. The calculations can certainly act as a guide for interpreting the near edge structure in terms of both molecular orbitals and various features in the band structure. It might also be possible to correlate the magnitude of near edge fine structure features with charge transfer.

\section{Acknowledgements.}

We should like to thank T. Manoubi, Orsay; K. Krishnan, C. Echer, Berkeley; and F. Hofer, Graz for making available experimental spectra. We should also like to acknowledge discussions with C. Colliex, W.M. Skiff, R. Brydson and O. Sankey which have lead to many of the ideas of this paper. Partial financial support from the NSF Facility for HREM (DMR 86 11609) is also acknowledged. 


\section{References}

[1] Durham P.J., Pendry J.B. and Hodges C.H., Computer Phys. Commun. 25 (1982) 193.

[2] HeRman F. and SkIllman S., Atomic Structure Calculations (Prentice Hall) 1963.

[3] MATTHEIS L.F., Phys. Rev. 134 (1964) A970.

[4] FuJIKAWA T., J. Phys. Soc. Jpn 52 (1983) 4001.

[5] SLATER J.C. and JOHNSON K.H., Phys. Rev. B5 (1972) 844.

[6] Lindner T., SAuer H., Engel W. and KaMBE K., Phys. Rev. B33 (1986) 22.

[7] Oizumi H., Fujikawa T., Ohashi M., MaEZawa H. and NAKaI S., J. Phys. Soc. Jpn 54 (1985) 4027.

[8] TAFTO J. and ZHU J., Ultramicroscopy 9 (1982) 349.

[9] Williams A.R., Kubler J. and GelatT C.D., Phys. Rev. B19 (1979) 6094.

[10] BLAHA P. and SCHWARZ K., Int. J. Quantum Chem. 23 (1983) 1535.

[11] JANSEN R.W. and SANKEY O.F., Phys. Rev. B36 (1987) 6520.

[12] HOHENBERG P. and KOHN W., Phys. Rev. 136 (1964) B864.

[13] WENG X., REZ P. and SANKEY O.F., Phys. Rev. B40 (1989) 5694.

[14] Weng X., MA H. and Rez P., Phys. Rev. B40 (1989) 4175.

[15] Weng X., ReZ P. and BATSON P.E., Solid State Commun. 74 (1990) 1013.

[16] CARSON R.D. and SCHNATTERLY S.E., Phys. Rev. Lett. 59 (1987) 319.

[17] Leapman R.D., FeJeS P.L. and SilcoX J., Phys. Rev. B28 (1983) 2361. 